


\title{
OPEN Associations of maternal vitamin D, PTH and calcium with hypertensive disorders of pregnancy and associated adverse perinatal outcomes: Findings from the Born in Bradford cohort study
}

\author{
Gillian Santorelli $\mathbb{D}^{1}$, Donald Whitelaw ${ }^{2}$, Diane Farrar ${ }^{1}$, Jane West $\mathbb{D}^{1}$ \& Debbie A. Lawlor ${ }^{3,4}$
}

Vitamin D and parathyroid hormone (PTH) regulate mineral metabolism and are required to maintain calcium levels. Vitamin $D$ deficiency is common, particularly during pregnancy, and has been associated with hypertensive disorders of pregnancy. We sought to determine whether maternal 25(OH)D, PTH and calcium concentrations at 26 weeks gestation are associated with adverse outcomes of pregnancy and establish whether these differ by ethnicity. This study included 476 White British and 534 Pakistani origin mother-offspring pairs from the Born in Bradford cohort study. We used multinomial or logistic regression to explore the association between vitamin $\mathrm{D}, \mathrm{PTH}$ and calcium with gestational hypertension (GH), pre-eclampsia (PE), caesarean section (CS), preterm birth (PTB) and small for gestational age (SGA). Pakistani women had lower $25(\mathrm{OH}) \mathrm{D}$ (median $13.0 \mathrm{vs} 36.0 \mathrm{nmol} / \mathrm{L}$ ), higher PTH (median 7.7 vs $3.3 \mathrm{pmol} / \mathrm{L}$ ) and similar calcium concentrations compared to White British women. In Pakistani women, higher concentrations of $25(\mathrm{OH}) \mathrm{D}$ were associated with a $60 \%$ increased odds of $\mathrm{GH}$, and a $37 \%$ reduced odds of $S G A ;$ PTH was associated with a $45 \%$ reduction in the odds of $\mathrm{GH}$. In White British women, each 1SD increase in calcium concentration was associated with a $34 \%$ increase in developing GH but a $33 \%$ reduction in the odds of PTB. Associations with PE and CS were consistent with the null. In conclusion, there are ethnic differences in the associations of $25(\mathrm{OH}) \mathrm{D}, \mathrm{PTH}$ and calcium with important perinatal outcomes. Future research would benefit from examining the associations of $25(\mathrm{OH}) \mathrm{D}, \mathrm{PTH}$ and calcium together with a range of perinatal outcomes in order to assess the riskbenefit action of each.

Low vitamin D levels are a global public health problem ${ }^{1}$. Vitamin D can be obtained from foods (in the form of $25(\mathrm{OH}) \mathrm{D}_{2}$ ) or dietary supplements, but the main source for most people is synthesised in the skin from sunlight exposure containing sufficient ultraviolet $\mathrm{B}(\mathrm{UVB})$ radiation $\left(25(\mathrm{OH}) \mathrm{D}_{3}\right)$. At latitudes above $37^{\circ} \mathrm{N}$, there is insufficient sunlight-induced vitamin D synthesis from October to late March; it is therefore essential to obtain vitamin D from dietary or supplementary sources during the winter months of northern countries, including the UK. People with darker skin are at particular risk of low vitamin D levels, and a number of studies have shown that South Asian's are known to have lower levels of 25-hydroxyvitamin D [25(OH)D] compared to the White population $^{2-4}$.

Vitamin D deficiency is commonly observed in pregnancy ${ }^{1}$ and has previously been associated with an increased risk of hypertensive disorders of pregnancy (gestational hypertension and pre-eclampsia) ${ }^{1,5-9}$, preterm

\footnotetext{
${ }^{1}$ Bradford Institute for Health Research, Temple Bank House, Bradford Royal Infirmary, Bradford, BD9 6RJ, UK. ${ }^{2}$ Department of Diabetes and Endocrinology, Bradford Royal Infirmary, Bradford, BD9 6RJ, UK. ${ }^{3} \mathrm{MRC}$ Integrated Epidemiology Unit at the University of Bristol, Rm OS11, Oakfield House, Oakfield Grove, Bristol, BS8 2 BN, UK. ${ }^{4}$ Bristol NIHR Biomedical Research Centre, Bristol, UK. Correspondence and requests for materials should be addressed to G.S. (email: gillian.santorelli@bthft.nhs.uk)
} 
birth $^{10-14}$, small for gestational age (SGA) infants ${ }^{14-21}$ and caesarean birth ${ }^{22-24}$. Together with parathyroid hormone (PTH), vitamin D is required for the regulation of calcium ${ }^{25}$; however, despite their close interactions, the association between them and adverse perinatal outcomes is still not fully understood. Although calcium supplementation during pregnancy seems to reduce the risk of high blood pressure, pre-eclampsia and preterm birth $^{26}$, the effects of vitamin D supplementation are less clear. A recent systematic review found evidence of an increase in mean birth weight and a reduced risk of SGA in women receiving vitamin D supplementation; however, no associations with other maternal and neonatal outcomes were observed and consequently the evidence was deemed insufficient to guide practice ${ }^{16}$. To our knowledge no studies have examined the combined associations between vitamin D, PTH and calcium levels with hypertensive disorders of pregnancy (HDP) and associated adverse perinatal outcomes or explored whether any of these associations differ between ethnic groups. Given the known ethnic differences in distributions of pregnancy circulating $25(\mathrm{OH}) \mathrm{D}^{27,28}$, which is a biomarker for vitamin D status, it is important to establish whether ethnicity influcences associations of $25(\mathrm{OH}) \mathrm{D}, \mathrm{PTH}$ and calcium with perinatal outcomes. This information could help determine whether thresholds for defining categories of vitamin $\mathrm{D}$ deficiency should differ by ethnicity.

The aims of this study are therefore to (a) determine whether maternal circulating $25(\mathrm{OH}) \mathrm{D}, \mathrm{PTH}$ and calcium concentrations are associated with gestational hypertension and pre-eclampsia and associated adverse perinatal outcomes (caesarean section, preterm birth and small for gestational age) in White British and Pakistani women, and (b) establish whether the magnitude or direction of associations between $25(\mathrm{OH}) \mathrm{D}, \mathrm{PTH}$ and calcium with these outcomes differ between White British and Pakistani women.

\section{Methods}

Born in Bradford ( $\mathrm{BiB})$ is a longitudinal multi-ethnic cohort study that was established to examine how genetic, nutrition, environmental, behavioural and social factors impact on health and development during childhood, and subsequently adult life $\mathrm{e}^{29}$. Bradford, located at $53.8^{\circ} \mathrm{N}$, is the $6^{\text {th }}$ largest city in the UK, is ethnically diverse, and has high levels of socio-economic deprivation. Women were recruited to $\mathrm{BiB}$ at approximately 26 weeks gestation when they attended for oral glucose tolerance testing (OGTT), performed as part of a universal screening programme for gestational diabetes. At recruitment, participants gave informed consent, height and weight were measured and an interview-administered baseline questionnaire completed. A total of 12,450 mothers were recruited across 13,773 pregnancies between March 2007 and December 2010, resulting in 13,858 births. Ethics approval was granted by Bradford National Health Service Research Ethics Committee (ref 07/H1302/112) on 10 March 2008 , and all research was performed in accordance with relevant guidelines/regulations. A subsample of 1,498 women who attended for assessment between September 2008 and March 2009 consented to have additional blood samples analysed to assess $25(\mathrm{OH}) \mathrm{D}, \mathrm{PTH}$ and calcium concentrations. We have previously reported findings from this cohort regarding associations between these variables and gestational diabetes, which suggested that whilst an association with $25(\mathrm{OH}) \mathrm{D}$ and $\mathrm{PTH}$ was not indicated, the association with calcium warranted further investigation $^{30}$. Women were excluded from the present analyses if $25(\mathrm{OH}) \mathrm{D}, \mathrm{PTH}$ and calcium levels were not measured $(n=26)$, perinatal outcomes were not reported $(n=18)$ and the baseline questionnaire was not completed $(n=155)$. Women who had a stillbirth $(n=10)$, a multiple birth $(n=20)$ or where the type of birth (singleton or multiple) was unknown $(n=19)$, or who were from an ethnic background other than White British or Pakistani $(n=240)$ were also excluded. The final sample size was 1010 (476 White British and 534 Pakistani; see Fig. 1).

Outcome measures. We assessed associations of maternal circulating $25(\mathrm{OH}) \mathrm{D}, \mathrm{PTH}$ and calcium (measured at 26-28 weeks gestation) with: (1) HDP: gestational hypertension (defined as new onset elevated blood pressure after 20 weeks gestation where SPB $\geq 140$ and/or DBP $\geq 90$ on two or more occasions), and pre-eclampsia (defined as gestational hypertension with concurrent significant proteinurea $\geq 1+$ ); (2) caesarean section; (3) preterm birth (defined as gestational age at birth $<37$ weeks); and (4) small for gestational age (SGA), based on the 2011 UK-WHO charts ${ }^{31}$.

Mode of birth (vaginal or caesarean section), birthweight and gestational age were extracted from the hospital's electronic maternity record system. Blood pressure measurements and levels of proteinurea during pregnancy were extracted from the maternity notes.

Exposures. Our exposures were maternal pregnancy concentrations of serum $25(\mathrm{OH}) \mathrm{D}$, plasma PTH and serum calcium. Fasting venous samples were taken at the time of the OGTT (median 26.3 weeks of gestation (IQR 26.0, 26.9).

Serum $25(\mathrm{OH}) \mathrm{D}$ samples were immediately frozen at $-80^{\circ} \mathrm{C}$ and analysed within 12 months with no interim thaw cycles. Serum samples were deproteinized using zinc sulphate and acetonitrile. We used deuterated $25(\mathrm{OH})$ $\mathrm{D}_{3}$ [hexadeuterium-25(OH) $\mathrm{D}_{3}$ ] as an internal standard in all analyses. Chromatographic separation was achieved on a Waters Sun Fire C18 column, and detection of $25(\mathrm{OH}) \mathrm{D}_{2}$ and $25(\mathrm{OH}) \mathrm{D}_{3}$ was achieved using Waters Micromass Quattro Ultima Platinum Mass Spectrometer. Between-batch coefficients of variation (CoV) were $5-8 \%$ and $4-6 \%$ for $25(\mathrm{OH}) \mathrm{D}_{2}$ and $25(\mathrm{OH}) \mathrm{D}_{3}$ respectively. As concentrations of $25(\mathrm{OH}) \mathrm{D}_{2}$ were generally very low, total $25(\mathrm{OH}) \mathrm{D}$ was calculated by summing $25(\mathrm{OH}) \mathrm{D}_{2}$ and $25(\mathrm{OH}) \mathrm{D}_{3}$ and is presented as nmol/L.

Plasma PTH and serum calcium were analysed promptly at Bradford Royal Infirmary (BRI). Chemiluminescence immunoassay was used to measure intact PTH with a manufacturer's quoted coefficients of variation of $7.8 \%$ at $4.3 \mathrm{pmol} / \mathrm{L}$ and a detection range of 0.265 to $201 \mathrm{pmol} / \mathrm{L}$. Calcium concentrations were measured using a colorimetric assay and were adjusted for albumin; values are presented as $\mathrm{mmol} / \mathrm{L}$.

Confounders. We adjusted our analyses for a number of confounders with 25(OH)D levels that have been identified by previous research. Information on maternal age, ethnicity, mother's highest educational qualifications (defined as less than A-level and A-level and above. A-levels are subject-specific qualifications taken by 


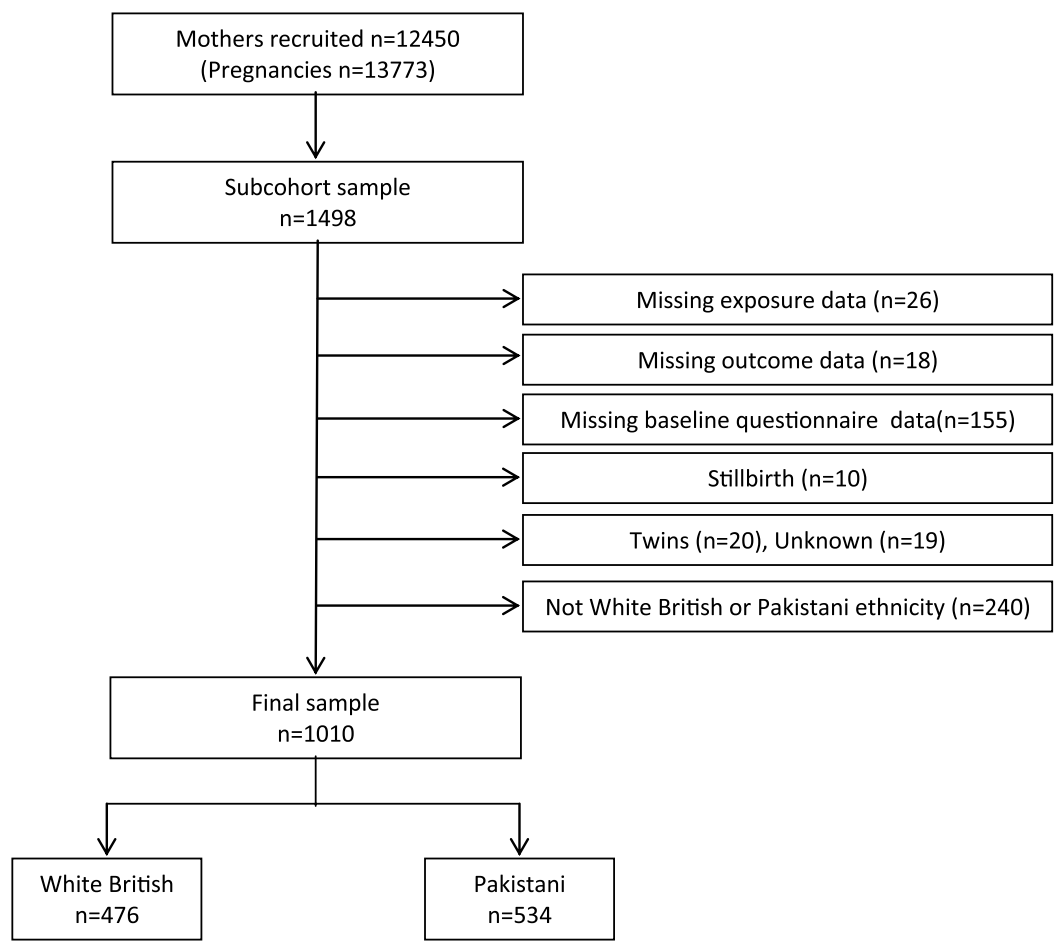

Figure 1. Study flow chart.

students aged 16-18 in England, Wales and Northern Ireland, and are the main school leaving qualifications in these countries) and in receipt of means-tested benefits (which aim to give extra support to individuals who can demonstrate that their income and capital are below a certain level) as proxy measures for socio-economic position, smoking during pregnancy, vitamin D supplementation (at least once a week) and physical activity (estimated using the General Practice Physical Activity Questionnaire (GPPAQ) ${ }^{32}$ ) were obtained from the baseline questionnaire. Early pregnancy BMI was derived from maternal height and weight measured at pregnancy booking ( $\sim 12$ weeks gestation). Sunshine exposure was estimated in the three months before the samples were taken using data from the Bradford station of the UK meteorological office ${ }^{33,34}$.

Statistical analysis. The distribution of outcomes and risk factors for both ethnic groups are presented as frequency (percentage), mean (SD) or median (IQR). Associations of $25(\mathrm{OH}) \mathrm{D}, \mathrm{PTH}$ and calcium with gestational hypertension, pre-eclampsia and associated adverse outcomes were assessed using multinomial or logistic regression with the exposure as a continuous variable. Two models were considered for each outcome: model 1 was unadjusted, and model 2 was adjusted for the confounders described above. The physical activity questionnaire has four categories: inactive, moderately inactive, moderately active and active; however, due to low numbers in the 'active' category, this was merged with 'moderately active' for the analysis. Associations between the exposures and outcomes are presented as odds ratios per 1 standard deviation (SD) increase. The analysis was done for all women, and for White British and Pakistani women separately and tested for any differences in associations between the ethnic groups (i.e. tested for an interaction between ethnicity and exposure). All analyses were performed using STATA/SE software (Stata/SE 13.1 for Windows, StataCorp LP, College Station, TX, USA).

Ethics approval. Ethics approval was granted by Bradford National Health Service Research Ethics Committee (ref 07/H1302/112) on 10 March 2008.

\section{Results}

Overall and ethnic differences in maternal exposures with gestational hypertension, pre-eclampsia and associated adverse perinatal outcomes. Table 1 shows the distribution of outcomes, exposures and maternal characteristics. Compared to White British women, Pakistani women were slightly older (27.6 vs 26.4 years), had a lower BMI (25.2 vs $\left.27.0 \mathrm{~kg} / \mathrm{m}^{2}\right)$, were less likely to smoke during pregnancy (3.8\% vs $\left.34.2 \%\right)$, were more likely to be in receipt of means-tested benefits (47.2 vs $37.0 \%)$ and reported lower physical activity levels ( $93.3 \%$ vs $76.0 \%$ inactive or moderately inactive). On average, Pakistani women had lower median levels of 25(OH)D (13.0 vs $\left.36.0 \mathrm{nmol} / \mathrm{mL}^{2}\right)$ and higher levels of PTH (7.9 vs $\left.3.3 \mathrm{pmol} / \mathrm{L}^{2}\right)$ than White British women; both groups had similar distributions of calcium (see Fig. 2). A greater proportion of White British women developed gestational hypertension ( $12.0 \%$ vs $5.4 \%)$ and pre-eclampsia (3.4\% vs $1.7 \%)$, and had a caesarean $(22.5 \%$ vs $19.9 \%)$ or preterm birth (6.5\% vs $4.5 \%)$ compared to Pakistani women. Conversely, delivery of a small for gestational age baby was more common in Pakistani women (19.9\% vs $8.6 \%)$. 


\begin{tabular}{|c|c|c|c|}
\hline Maternal characteristics and perinatal outcomes & All $\mathrm{N}=1010$ & White British $\mathrm{N}=476$ & Pakistani N $=534$ \\
\hline Age (years) ${ }^{*}$ & $27.0 \pm 5.5$ & $26.4 \pm 6.3$ & $27.6 \pm 5.3$ \\
\hline BMI at 12 weeks gestation ${ }^{*}$ & $26.0 \pm 5.8$ & $27.0 \pm 6.2$ & $25.2 \pm 5.3$ \\
\hline \multicolumn{4}{|l|}{ Smoked during pregnancy } \\
\hline No & $827(81.9)$ & $313(65.8)$ & $514(96.3)$ \\
\hline Yes & $183(18.1)$ & $163(34.2)$ & $20(3.8)$ \\
\hline \multicolumn{4}{|l|}{ In receipt of means-tested benefits } \\
\hline No & $582(57.6)$ & $300(62.4)$ & $282(52.8)$ \\
\hline Yes & $428(42.4)$ & $176(37.0)$ & $252(47.2)$ \\
\hline \multicolumn{4}{|l|}{ Maternal education } \\
\hline Less than A level & $627(62.1)$ & $304(63.9)$ & $323(60.5)$ \\
\hline A level and above & $383(37.9)$ & $172(36.1)$ & $211(39.5)$ \\
\hline \multicolumn{4}{|l|}{ Physical activity level } \\
\hline Inactive & $668(66.1)$ & $242(50.8)$ & $426(79.8)$ \\
\hline Moderately inactive & $181(19.0)$ & $120(25.2)$ & $72(13.5)$ \\
\hline Moderately active & $94(9.3)$ & $70(14.7)$ & $24(4.5)$ \\
\hline Active & $56(5.5)$ & $44(9.2)$ & $12(2.3)$ \\
\hline \multicolumn{4}{|l|}{ Vitamin D supplementation } \\
\hline No & $835(82.7)$ & $389(81.7)$ & $446(83.5)$ \\
\hline Yes & $175(17.3)$ & $87(18.3)$ & $88(16.5)$ \\
\hline Sunshine, hours per $3 \mathrm{mths}^{\dagger}$ & $248.9(161.9-315.7)$ & $258.5(164.2,322.6)$ & $237.7(159.5,308.9)$ \\
\hline $25(\mathrm{OH}) \mathrm{D}, \mathrm{nmol} / \mathrm{mL}^{\dagger}$ & $22.6(12.0-40.8)$ & $36.0(26.0-55.5)$ & $13.0(8.8-20.3)$ \\
\hline \multicolumn{4}{|l|}{ Vitamin D status } \\
\hline Deficient & $598(61.5)$ & $160(34.8)$ & $438(85.4)$ \\
\hline Insufficient & $196(20.1)$ & $152(33.0)$ & $44(8.6)$ \\
\hline Adequate & $179(18.4)$ & $148(32.2)$ & $31(6.0)$ \\
\hline $\mathrm{PTH}, \mathrm{pmol} / \mathrm{L}^{\dagger}$ & $5.0(3.2-8.2)$ & $3.3(2.4-4.5)$ & $7.9(5.5-11.3)$ \\
\hline Calcium, $\mathrm{mmol} / \mathrm{L}^{\dagger}$ & $2.25(2.20-2.29)$ & $2.25(2.22-2.30)$ & $2.24(2.19-2.28)$ \\
\hline Gestational hypertension & $86(8.5)$ & $57(12.0)$ & $29(5.4)$ \\
\hline Pre-eclampsia & $25(2.5)$ & $16(3.4)$ & $9(1.7)$ \\
\hline Caesarean birth & $213(21.1)$ & $107(22.5)$ & $106(19.9)$ \\
\hline Preterm birth ( <37 wks) & $55(5.5)$ & $31(6.5)$ & $24(4.5)$ \\
\hline Small for gestational age & $147(14.6)$ & $41(8.6)$ & $106(19.9)$ \\
\hline
\end{tabular}

Table 1. Maternal characteristics and perinatal outcomes for all pregnancies and by ethnic group (White British and Pakistani). Values are n (\%) unless otherwise indicated. $*$ Mean (SD); ${ }^{\dagger}$ Median (IQR).

Associations between 25(OH)D, PTH and calcium with gestational hypertension, pre-eclampsia and associated adverse perinatal outcomes. Adjusted (Model 2) associations of each exposure with outcomes in White British and Pakistani mother-offspring pairs are shown in Table 2, and Figs 3 and 4, with the unadjusted (Model 1) results in the supplementary material. In the adjusted analyses, higher concentrations of $25(\mathrm{OH}) \mathrm{D}$ in Pakistani women were associated with a $60 \%$ increased odds of developing gestational hypertension, whereas higher PTH levels were associated with a $45 \%$ relative reduction, and there was evidence of a statistical interaction between the ethnic groups ( $p<0.01$ and $p=0.10$ respectively). 25(OH)D was also associated with a reduced odds of SGA and higher calcium concentrations were associated with gestational hypertension but a reduced odds of preterm birth in White British but not Pakistani women; however, there was no strong statistical support for any ethnic differences, with $p$-values for interaction with ethnicity being $\geq 1.0$. Associations with caesarean delivery were broadly consistent with the null.

\section{Discussion}

Main findings. We found that at 26 weeks gestation, White British women had markedly higher levels of 25(OH)D levels, lower PTH and similar calcium levels compared to Pakistani women. We found evidence for ethnic differences in associations between maternal 25(OH)D and PTH with gestational hypertension. Where point estimates differed between the two groups, the directions of these with respect to clinical outcomes were inconsistent. For example, our results suggest higher average 25(OH)D could be detrimental to Pakistani women by increasing their risk of gestational hypertension but beneficial in reducing their risk of SGA. Higher calcium concentrations were associated with an increased risk of gestational hypertension but reduced the risk of preterm birth in White British women.

Strengths and limitations. The strengths of our study include its prospective bi-ethnic cohort design, direct measurement of 25(OH)D, PTH and calcium levels and detailed information on a number of potential confounders. Limitations are that due to overall small numbers, and hence relatively few cases for the outcomes 

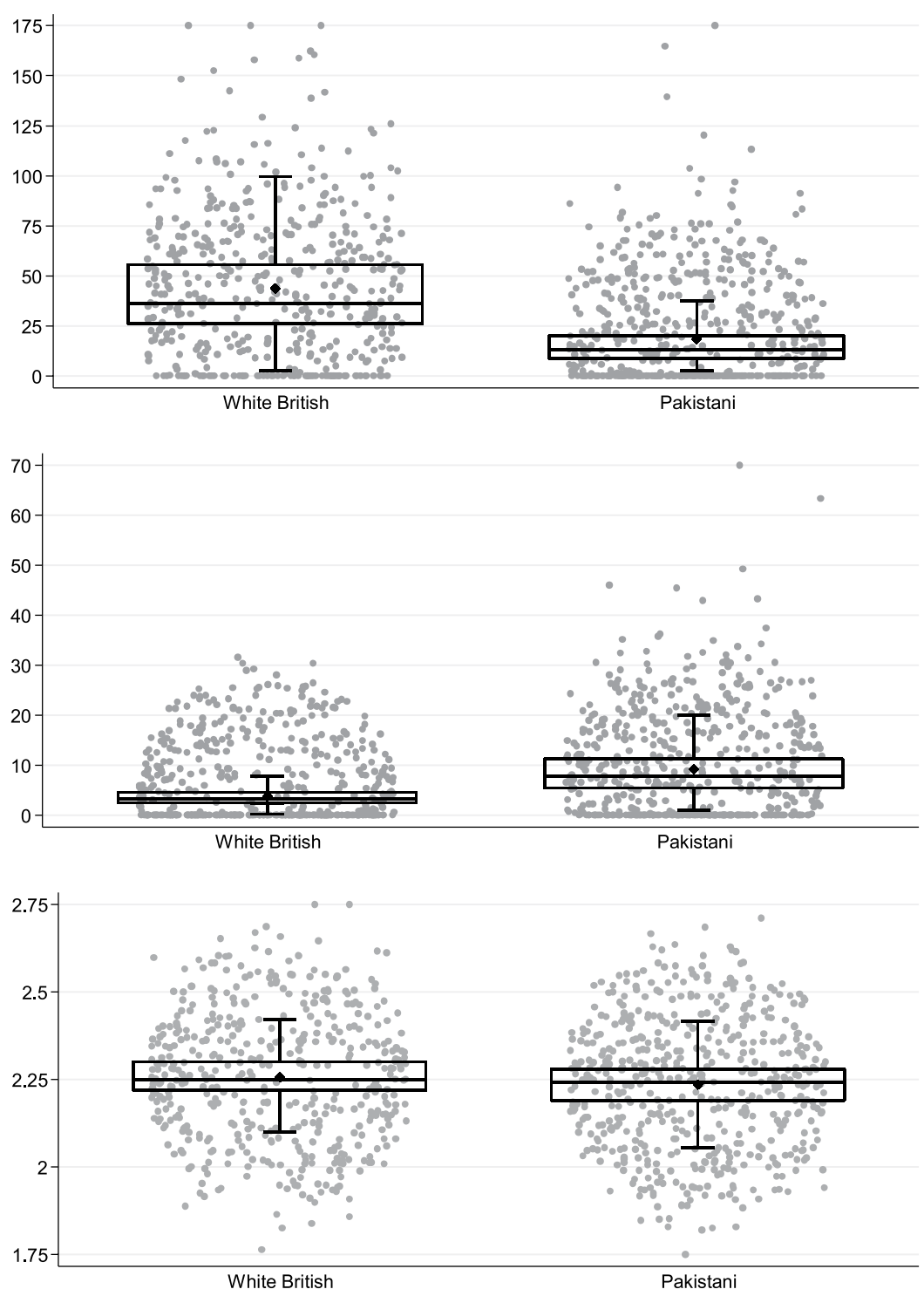

Figure 2. Box and scatter plots of maternal circulating $25(\mathrm{OH}) \mathrm{D}, \mathrm{PTH}$ and calcium concentrations in White British and Pakistani women.

examined, we have limited statistical power, especially when considering ethnic differences, however our study is able to show potential areas where investigation should be directed. Related to the relatively small sample sizes we were unable to explore possible non-linear associations of $25(\mathrm{OH}) \mathrm{D}, \mathrm{PTH}$ and calcium with our outcomes. It is known that concentrations of $25(\mathrm{OH}) \mathrm{D}$ fluctuate over time; however we were only able to assess it once, at around 26 weeks gestation. It would have been desirable to have also had data at earlier and later time-points in order to evaluate change over time, as it is possible that concentrations at different stages of fetal development or that change during pregnancy is important in the development of adverse perinatal outcomes. Due to a lack of diet-related data, we were not able to adjust for this in our analyses. However, we have directly measured total plasma $25(\mathrm{OH}) \mathrm{D}$ concentration, and have accounted for sunshine exposure and vitamin $\mathrm{D}$ supplementation in the analyses. As few foods are naturally rich in vitamin $\mathrm{D}$, it is unlikely that including diet in our analyses would have had an impact. Our results are from White British and Pakistani origin women resident in the UK and may therefore not be generalisable to other White or South Asian ethnic groups. However, in stratifying our analyses by ethnic group we were able to observe the effect of the exposures on outcomes in the majority ethnic group in the UK (White British) and in Pakistani-origin women, who account for a significant proportion of the population in a number of UK towns and cities.

Interpretation. There is growing evidence that low levels of $25(\mathrm{OH}) \mathrm{D}$ can adversely affect cardiometabolic health in adults including associations with both hypertension and insulin resistance ${ }^{35,36}$. In pregnancy, there is evidence to suggest an association between $25(\mathrm{OH}) \mathrm{D}$ and the pathophysiology of pre-eclampsia ${ }^{8}$. One recent study reported similar 25-hydroxyvitamin D3 levels in normotensive pregnant women and women with pre-eclampsia, but higher levels of vitamin D metabolites and impaired placental uptake of 25-hydroxyvitamin 


\begin{tabular}{|c|c|c|c|c|}
\hline \multirow[b]{2}{*}{ Outcome } & \multicolumn{3}{|c|}{$\mathrm{aOR}^{\dagger}(95 \% \mathrm{CI})$ for each outcome per $1 \mathrm{SD}$ of exposure } & \multirow[b]{2}{*}{$\mathbf{P}_{\text {Interaction }}{ }^{\ddagger}$} \\
\hline & All $(n=978)$ & $\begin{array}{l}\text { White British } \\
(n=462)\end{array}$ & $\begin{array}{l}\text { Pakistani } \\
(\mathrm{n}=516)\end{array}$ & \\
\hline \multicolumn{5}{|l|}{ HDP $^{*}$} \\
\hline \multicolumn{5}{|l|}{$25(\mathrm{OH}) \mathrm{D}$} \\
\hline Gestational hypertension & $1.2014(0.94-1.54)$ & $0.81(0.55-1.21)$ & $1.60(1.00-2.55)$ & $<0.001$ \\
\hline Pre-eclampsia & $1.33(0.91-1.94)$ & $1.18(0.69-1.99)$ & $1.88(0.78-4.50)$ & 0.58 \\
\hline \multicolumn{5}{|l|}{ PTH } \\
\hline Gestational hypertension & $0.55(0.37-0.83)$ & $0.96(0.43-2.14)$ & $0.55(0.30-1.01)$ & 0.10 \\
\hline Pre-eclampsia & $1.05(0.66-1.66)$ & $1.02(0.25-4.21)$ & $1.31(0.81-2.11)$ & 0.87 \\
\hline \multicolumn{5}{|l|}{ Calcium } \\
\hline Gestational hypertension & $1.35(1.08-1.21)$ & $1.34(1.01-1.76)$ & $1.17(0.78-1.76)$ & 0.70 \\
\hline Pre-eclampsia & $0.92(0.60-1.39)$ & $0.92(0.55-1.56)$ & $0.85(0.40-1.81)$ & 0.95 \\
\hline \multicolumn{5}{|l|}{ Caesarean birth } \\
\hline $25(\mathrm{OH}) \mathrm{D}$ & $1.02(0.86-1.21)$ & $0.97(0.76-1.25)$ & $1.17(0.83-1.66)$ & 0.31 \\
\hline PTH & $0.93(0.77-1.11)$ & $0.80(0.44-1.45)$ & $0.93(0.75-1.16)$ & 0.99 \\
\hline Calcium & $0.94(0.80-1.10)$ & $0.906(0.77-1.20)$ & $0.94(0.75-1.18)$ & 0.77 \\
\hline \multicolumn{5}{|l|}{ Preterm birth } \\
\hline $25(\mathrm{OH}) \mathrm{D}$ & $1.16(0.86-1.55)$ & $1.06(0.72-1.57)$ & $1.10(0.53-2.28)$ & 0.78 \\
\hline PTH & $1.11(0.86-1.45)$ & $1.73(0.71-4.18)$ & $1.07(0.85-1.54)$ & 0.60 \\
\hline Calcium & $0.84(0.63-1.12)$ & $0.67(0.44-1.01)$ & $1.07(0.69-1.68)$ & 0.12 \\
\hline \multicolumn{5}{|l|}{ Small for gestational age } \\
\hline $25(\mathrm{OH}) \mathrm{D}$ & $0.58(0.44-0.77)$ & $0.93(0.63-1.37)$ & $0.63(0.37-1.04)$ & 0.37 \\
\hline $\mathrm{PTH}$ & $1.30(1.10-1.53)$ & $0.59(0.21-1.68)$ & $1.14(0.95-1.37)$ & 0.31 \\
\hline Calcium & $1.08(0.90-1.30)$ & $1.24(0.88-1.74)$ & $1.11(0.89-1.40)$ & 0.67 \\
\hline
\end{tabular}

Table 2. Adjusted associations of maternal circulating 25(OH)D, PTH and calcium with hypertensive disorders of pregnancy (HDP) and adverse associated outcomes overall and stratified by ethnicity. *Hypertensive disorders of pregnancy; normotensive is the reference category. ${ }^{\dagger}$ Adjusted for maternal age, BMI, highest educational attainment, smoking status in pregnancy, in receipt of benefits, physical activity levels, hours of sunshine and vitamin D supplementation. ${ }^{\ddagger}$ P-value for interaction with ethnicity.

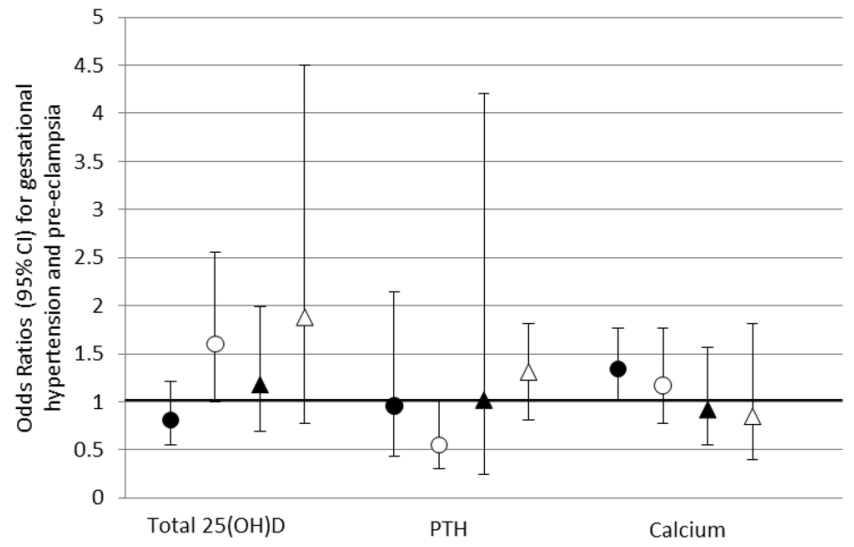

Figure 3. Adjusted odds ratios with $95 \%$ CI for associations between a 1 SD increase in maternal circulating 25(OH)D, PTH and calcium with hypertensive disorders of pregnancy. Gestational hypertension: White British women $\boldsymbol{\Delta}$ Pakistani women. Pre-eclampsia: $\bigcirc$ White British women $\triangle$ Pakistani women.

D3 in women with pre-eclampsia ${ }^{37}$, suggesting vitamin D metabolism may influence physiological processes in a number of ways.

Pre-eclampsia is associated with increased maternal and infant mortality and morbidity therefore interventions to ameliorate these risks urgently required. Vitamin D supplementation to improve outcomes including reducing the risk of pre-eclampsia continues to attract investigation. A recent systematic review included 43 trials and identified a further 35 registered trials, unfortunately only eight of the 43 included trials were considered at low risk of bias ${ }^{16}$. Conflicting with data from previous observational studies showing an inverse relationship with vitamin D levels and pre-eclampsia, we demonstrated a positive association and the review of Vitamin D supplementation found no evidence of an effect on risk of pre-eclampsia or gestational hypertension. Reasons for inconsistencies between trials and observational studies investigating associations are unclear, but may include: 

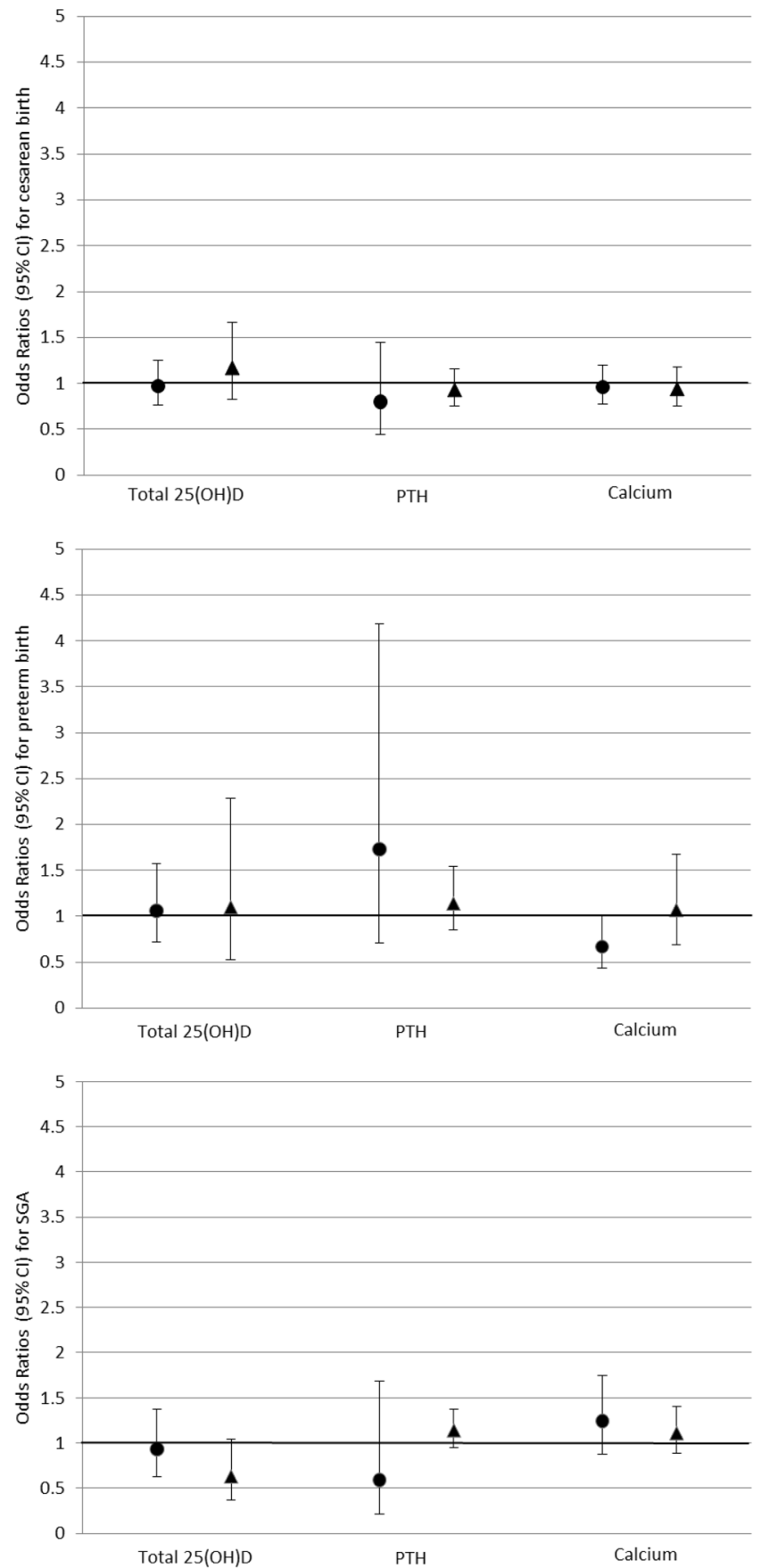

Figure 4. Adjusted odds ratios with $95 \%$ CI for associations between a 1 SD increase in maternal circulating 25(OH)D, PTH and calcium with caesarean section, preterm birth and small for gestational age. White British women $\mathbf{A}$ Pakistani women.

the possibility that there are different effects associated with naturally occurring vitamin $\mathrm{D}$ synthesised from sunlight compared to vitamin D taken as a supplement; different effects from natural or artificial vitamin D metabolites; heterogeneity of included trials in the review and variation in the definition of pre-eclampsia across trials or effects from variables not measured in observational studies. Our study and the findings of two other reviews share similarities in that we found higher concentrations of $25(\mathrm{OH}) \mathrm{D}$ reduced the risk of SGA ${ }^{16,17}$ and an increase in mean birth weight ${ }^{16}$ with supplementation. Low vitamin D may lead to low calcium levels which may in turn lead to increased vasoconstriction and hypertension by stimulating PTH or renin release, and calcium has previously been shown to be inversely related to pre-eclampsia ${ }^{38}$. Strong evidence from high quality trials suggests a beneficial effect on high blood pressure and pre-eclampsia from calcium supplementation (at least $1 \mathrm{~g} /$ day) in pregnancy, but an increase in the risk of HELLP (haemolysis, elevated liver enzymes and low platelets) syndrome; the authors suggest this latter inconsistent finding may result from the control of blood pressure without treatment of the underlying pre-eclamptic process ${ }^{26}$. Conversely we found a positive association between calcium and 
gestational hypertension, which may be a result of small numbers and consequently few events but may also be due to the relatively small change in each SD of calcium in unsupplemented women in our study compared to the large differences in calcium levels from supplementation.

\section{Conclusion}

Our findings indicate ethnic differences exist in the associations between $25(\mathrm{OH}) \mathrm{D}$, PTH and calcium with HDP and associated adverse perinatal outcomes, and that the directions of these associations were inconsistent with respect to clinical importance. The potential combination for benefit and harm for different outcomes highlights the importance of examining several related exposure and outcomes in one study.

\section{Data Availability Statement}

Data can be made available upon request.

\section{References}

1. Palacios, C. \& Gonzalez, L. Is vitamin D deficiency a major global public health problem? The Journal of steroid biochemistry and molecular biology 144 Pt A, 138-145, https://doi.org/10.1016/j.jsbmb.2013.11.003 (2014).

2. Darling, A. L. et al. Vitamin D deficiency in UK South Asian Women of childbearing age: a comparative longitudinal investigation with UK Caucasian women. Osteoporosis international: a journal established as result of cooperation between the European Foundation for Osteoporosis and the National Osteoporosis Foundation of the USA 24, 477-488, https://doi.org/10.1007/s00198-012-1973-2 (2013).

3. Kift, R. et al. Lifestyle factors including less cutaneous sun exposure contribute to starkly lower vitamin D levels in U.K. South Asians compared with the white population. The British journal of dermatology 169, 1272-1278, https://doi.org/10.1111/bjd.12518 (2013).

4. Patel, J. V. et al. Vitamin D deficiency amongst minority ethnic groups in the UK: a cross sectional study. International journal of cardiology 167, 2172-2176, https://doi.org/10.1016/j.ijcard.2012.05.081 (2013).

5. Bodnar, L. M. et al. Maternal vitamin D deficiency increases the risk of preeclampsia. The Journal of clinical endocrinology and metabolism 92, 3517-3522, https://doi.org/10.1210/jc.2007-0718 (2007).

6. Burris, H. H. et al. Vitamin D status and hypertensive disorders in pregnancy. Annals of epidemiology 24, 399-403 e391, https://doi. org/10.1016/j.annepidem.2014.02.001 (2014).

7. Hypponen, E. et al. Vitamin D and pre-eclampsia: original data, systematic review and meta-analysis. Annals of nutrition \& metabolism 63, 331-340, https://doi.org/10.1159/000358338 (2013).

8. Kawtar, N., Wafae, R., Saadia, J. \& Ouafa, M. Vitamin D and Pre-eclampsia. Gynecol Obstet 6 (2016)

9. Tabesh, M., Salehi-Abargouei, A., Tabesh, M. \& Esmaillzadeh, A. Maternal vitamin D status and risk of pre-eclampsia: a systematic review and meta-analysis. The Journal of clinical endocrinology and metabolism 98, 3165-3173, https://doi.org/10.1210/jc.2013-1257 (2013).

10. Amegah, A. K., Klevor, M. K. \& Wagner, C. L. Maternal vitamin D insufficiency and risk of adverse pregnancy and birth outcomes: A systematic review and meta-analysis of longitudinal studies. PLoS One 12, e0173605, https://doi.org/10.1371/journal. pone.0173605 (2017)

11. McDonnell, S. L. et al. Maternal 25(OH)D concentrations $>/=40 \mathrm{ng} / \mathrm{mL}$ associated with $60 \%$ lower preterm birth risk among general obstetrical patients at an urban medical center. PLoS One 12, e0180483, https://doi.org/10.1371/journal.pone.0180483 (2017).

12. Qin, L. L., Lu, F. G., Yang, S. H., Xu, H. L. \& Luo, B. A. Does Maternal Vitamin D Deficiency Increase the Risk of Preterm Birth: A Meta-Analysis of Observational Studies. Nutrients 8, https://doi.org/10.3390/nu8050301 (2016).

13. Tabatabaei, N. et al. Maternal Vitamin D Insufficiency Early in Pregnancy Is Associated with Increased Risk of Preterm Birth in Ethnic Minority Women in Canada. The Journal of nutrition, https://doi.org/10.3945/jn.116.241216 (2017).

14. Wei, S. Q., Qi, H. P., Luo, Z. C. \& Fraser, W. D. Maternal vitamin D status and adverse pregnancy outcomes: a systematic review and meta-analysis. The journal of maternal-fetal \& neonatal medicine: the official journal of the European Association of Perinatal Medicine, the Federation of Asia and Oceania Perinatal Societies, the International Society of Perinatal Obstet 26, 889-899, https://doi. org/10.3109/14767058.2013.765849 (2013).

15. Aghajafari, F. et al. Association between maternal serum 25-hydroxyvitamin D level and pregnancy and neonatal outcomes: systematic review and meta-analysis of observational studies. Bmj 346, f1 169, https://doi.org/10.1136/bmj.f1169 (2013).

16. Roth, D. E. et al. Vitamin D supplementation during pregnancy: state of the evidence from a systematic review of randomised trials. Bmj 359, j5237, https://doi.org/10.1136/bmj.j5237 (2017).

17. Bi, W. G. et al. Association Between Vitamin D Supplementation During Pregnancy and Offspring Growth, Morbidity, and Mortality: A Systematic Review and Meta-analysis. JAMA pediatrics, https://doi.org/10.1001/jamapediatrics.2018.0302 (2018).

18. Bodnar, L. M. et al. Maternal serum 25-hydroxyvitamin D concentrations are associated with small-for-gestational age births in white women. The Journal of nutrition 140, 999-1006, https://doi.org/10.3945/jn.109.119636 (2010).

19. Burris, H. H. et al. Plasma 25-hydroxyvitamin D during pregnancy and small-for-gestational age in black and white infants. Annals of epidemiology 22, 581-586, https://doi.org/10.1016/j.annepidem.2012.04.015 (2012).

20. Chen, Y. H. et al. Maternal vitamin D deficiency during pregnancy elevates the risks of small for gestational age and low birth weight infants in Chinese population. The Journal of clinical endocrinology and metabolism 100, 1912-1919, https://doi.org/10.1210/ jc.2014-4407 (2015).

21. Leffelaar, E. R., Vrijkotte, T. G. \& van Eijsden, M. Maternal early pregnancy vitamin D status in relation to fetal and neonatal growth: results of the multi-ethnic Amsterdam Born Children and their Development cohort. The British journal of nutrition 104, 108-117, https://doi.org/10.1017/S000711451000022X (2010).

22. Merewood, A., Mehta, S. D., Chen, T. C., Bauchner, H. \& Holick, M. F. Association between vitamin D deficiency and primary cesarean section. The Journal of clinical endocrinology and metabolism 94, 940-945, https://doi.org/10.1210/jc.2008-1217 (2009).

23. Rodriguez, A. et al. Associations of maternal circulating 25-hydroxyvitamin D3 concentration with pregnancy and birth outcomes. BJOG: an international journal of obstetrics and gynaecology 122, 1695-1704, https://doi.org/10.1111/1471-0528.13074 (2015).

24. Scholl, T. O., Chen, X. \& Stein, P. Maternal vitamin D status and delivery by cesarean. Nutrients 4, 319-330, https://doi.org/10.3390/ nu4040319 (2012).

25. Holick, M. F. \& C. C. T. Vitamin D deficiency: a worldwide problem with health consequences. Am J clin nutrition 87, 1080S-1086S (2008).

26. Hofmeyr, G. J., Lawrie, T. A., Atallah, Á. N., Duley, L. \& Torloni, M. R. Calcium supplementation during pregnancy for preventing hypertensive disorders and related problems. Cochrane Database Syst Revs (2014).

27. Yu, C. K., Sykes, L., Sethi, M., Teoh, T. G. \& Robinson, S. Vitamin D deficiency and supplementation during pregnancy. Clinical endocrinology 70, 685-690, https://doi.org/10.1111/j.1365-2265.2008.03403.x (2009).

28. Eggemoen, Å. R. et al. Vitamin D deficiency and supplementation in pregnancy in a multiethnic population-based cohort. $B M C$ Pregnancy Childbirth 16, 7 (2016). 
29. Wright, J. et al. Cohort Profile: the Born in Bradford multi-ethnic family cohort study. International journal of epidemiology 42 , 978-991, https://doi.org/10.1093/ije/dys112 (2013).

30. Whitelaw, D. C. et al. Associations of circulating calcium and 25-hydroxyvitamin D with glucose metabolism in pregnancy: a crosssectional study in European and South Asian women. The Journal of clinical endocrinology and metabolism 99, 938-946, https://doi. org/10.1210/jc.2013-2896 (2014).

31. Cole, T. J., Williams, A. F., Wright, C. M. \& Group, R. G. C. E. Revised birth centiles for weight, length and head circumference in the UK-WHO growth charts. Annals of human biology 38, 7-11, https://doi.org/10.3109/03014460.2011.544139 (2011).

32. Department of Health. General Practise Physical Activity Questionnaire (2009).

33. Met Office, http:/www.metoffice.gov.uk/climate/uk/stationdata/bradforddata.

34. Sayers, A., Tilling, K., Boucher, B. J., Noonan, K. \& Tobias, J. H. Predicting ambient ultraviolet from routine meteorological data; its potential use as an instrumental variable for vitamin D status in pregnancy in a longitudinal birth cohort in the UK. International journal of epidemiology 38, 1681-1688, https://doi.org/10.1093/ije/dyp237 (2009).

35. Forman, J. P. et al. Plasma 25-hydroxyvitamin D levels and risk of incident hypertension. Hypertension 49, 1063-1069, https://doi. org/10.1161/HYPERTENSIONAHA.107.087288 (2007).

36. Forouhi, N. G., Luan, J., Cooper, A., Boucher, B. J. \& Wareham, N. J. Baseline serum 25-hydroxy vitamin d is predictive of future glycemic status and insulin resistance: the Medical Research Council Ely Prospective Study 1990-2000. Diabetes 57, 2619-2625, https://doi.org/10.2337/db08-0593 (2008).

37. Tamblyn, J. A. et al. Dysregulation of maternal and placental vitamin D metabolism in preeclampsia. Placenta 50, 70-77, https://doi. org/10.1016/j.placenta.2016.12.019 (2017).

38. Belizán, J. M., Villar, J. \& Repke, J. The relationship between calcium intake and pregnancy-induced hypertension: Up-to-date evidence. Am J Obstet Gynecol 158, 898-902, https://doi.org/10.1016/0002-9378(88)90091-9 (1988).

\section{Acknowledgements}

Born in Bradford is only possible because of the enthusiasm and commitment of all the children and parents in Born in Bradford. We thank all the participants, health professionals and researchers who have made Born in Bradford happen. BiB receives core infrastructure funding from the Wellcome Trust (WT101597M) and a joint grant from the UK Medical Research Council (MRC) and Economic and Social Science Research Council (ESRC) (MR/N024397/1). Laboratory analysis of plasma vitamin D, PTH and calcium levels was supported by a Diabetes UK project grant (08/0003672). DF is supported by a Post-doctoral NIHR fellowship. DAL works in a unit that received UK MRC funding (MC_UU_10213/5) and is a NIHR senior investigator (NF-SI-0611-10196).

\section{Author Contributions}

Donald Whitelaw (D.W.) and Gillian Santorelli (G.S.) conceived the study idea. G.S. and D.A. Lawlor (D.A.L.) wrote the analysis plan. G.S. analysed the data and wrote the first draft of the manuscript. G.S., D.W., Diane Farrar (D.F.), Jane West (J.W.) and D.A.L. contributed to further drafts and have read and approved the final manuscript.

\section{Additional Information}

Supplementary information accompanies this paper at https://doi.org/10.1038/s41598-018-37600-9.

Competing Interests: The authors declare no competing interests.

Publisher's note: Springer Nature remains neutral with regard to jurisdictional claims in published maps and institutional affiliations.

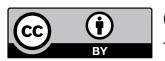

Open Access This article is licensed under a Creative Commons Attribution 4.0 International License, which permits use, sharing, adaptation, distribution and reproduction in any medium or format, as long as you give appropriate credit to the original author(s) and the source, provide a link to the Creative Commons license, and indicate if changes were made. The images or other third party material in this article are included in the article's Creative Commons license, unless indicated otherwise in a credit line to the material. If material is not included in the article's Creative Commons license and your intended use is not permitted by statutory regulation or exceeds the permitted use, you will need to obtain permission directly from the copyright holder. To view a copy of this license, visit http://creativecommons.org/licenses/by/4.0/.

(c) The Author(s) 2019 